\title{
Assessment of Urinary Albumin/Creatinine Ratio in Non-Obese Non-Diabetic Patients with Nonalcoholic Fatty Liver Disease
}

\author{
Ali Hussein Mohammed1*, Ahmed Sedky², Hisham A. Alghany Algahlan³, Amr M. Zaghloul ${ }^{4}$ \\ ${ }^{1}$ Department of Internal Medicine, Faculty of Medicine, Sohag University, Sohag, Egypt \\ ${ }^{2}$ Department of Clinical Pathology, Faculty of Medicine, Sohag University, Sohag, Egypt \\ ${ }^{3}$ Department of Diagnostic Radiology, Faculty of Medicine, Sohag University, Sohag, Egypt \\ ${ }^{4}$ Department of Tropical Medicine, Faculty of Medicine, Sohag University, Sohag, Egypt \\ Email: *ahussein79@yahoo.com
}

How to cite this paper: Mohammed, A.H. Sedky, A., Algahlan, H.A.A. and Zaghloul, A.M. (2020) Assessment of Urinary Albumin/Creatinine Ratio in Non-Obese NonDiabetic Patients with Nonalcoholic Fatty Liver Disease. Open Journal of Gastroenterology, 10, 61-71.

https://doi.org/10.4236/ojgas.2020.104007

Received: February 29, 2020

Accepted: April 10, 2020

Published: April 13, 2020

Copyright (c) 2020 by author(s) and Scientific Research Publishing Inc. This work is licensed under the Creative Commons Attribution International License (CC BY 4.0).

http://creativecommons.org/licenses/by/4.0/

\begin{abstract}
Nonalcoholic fatty liver disease (NAFLD) is a pathological condition seen as histological change ranging from simple steatosis to steatohepatitis, advanced fibrosis and liver disease among patients without significant alcohol consumption. Microalbuminuria which is defined as the urinary albumin excretion of $30-300 \mathrm{mg} / 24 \mathrm{~h}$ has been reported to be a risk factor for renal and cardiovascular disorders. It also has independent correlation to high mortality in diabetic and hypertensive patients. NAFLD is affecting non obese non diabetic individuals; Microalbuminuria is correlated to visceral adipose tissue even in non diabetic non obese patients with limited studies in this aspect. Microalbuminuria is considered as a risk factor for cardiovascular and chronic kidney disease. Aim of the work: To assess urinary albumin creatinine ratio in non-obese non-diabetic patients with nonalcoholic fatty liver disease. $\mathrm{Pa}$ tients and methods: Total number of 80 patients with NAFLD that were non diabetic non obese patients. Abdominal ultrasonography and laboratory investigations were done. Results: Eighty non-obese, non-diabetic subjects (32 women, 48 men) with the mean age of 50.9 were included in this study. The population of the study was classified into four groups according to ultrasonographic degrees of steatosis. Control Group (A), (No. 25) 31.25\% of total cases are reported as no fat accumulation in liver. Group B; No. $2126.25 \%$ of total cases are reported as had mild steatosis (NAFLD 1). Group C; No. 18 (22.5\%) of total cases and reported as had moderate fat accumulation (moderate steatosis). Group D; No. 16 (20\%) of total cases reported had severe fat accumulation (severe steatosis). Urinary albumin creatinine ratio also shows increase in its values with increasing in the degree of steatosis among differ-
\end{abstract}


ent groups which is highly statistically significant. Triglycerides, total cholesterol and LDL show also significant changes between groups as they are significantly increased in value as regard increasing in degree of steatosis, inversely noticed with HDL levels as it goes down with elevated degree of steatosis which is statistically significant. Discussion: The effects of fatty liver disease on renal functions have been evaluated in some studies; in this study we tried to evaluate the correlation between microalbuminuria and various degrees of steatosis in non-obese non diabetic patients; we found that NAFLD could be seen in non obese non diabetic individuals with special reference to other factors that may influence the progress of the disease such as hyperlipidemia with increased risk of microalbuminuria among those patients. Conclusion: After exclusion of type $2 \mathrm{DM}$ and obesity, we conclude that the presence and the severity of microalbuminuria are more apparent among NAFLD patients.

\section{Keywords}

Microalbuminuria, NAFLD, Chronic Kidney Disease

\section{Introduction}

Nonalcoholic fatty liver disease (NAFLD) is a pathological condition seen as histological changes ranging from simple steatosis to steatohepatitis, advanced fibrosis and liver disease among patients without significant alcohol consumption [1].

NAFLD is thought of as the effect of metabolic syndrome on the liver, as it is closely related to insulin resistance and oxidative stress [2] [3]. NAFLD patients are defined to have higher risk of cardiovascular and systemic disorders [4]. Patients with NAFLD have been reported to have an increased carotid artery wall thickness than those without NAFLD [1] [5].

In spite of the fact that obesity and diabetes mellitus are the two primary hazards for the advancement of NAFLD, non obese, non-diabetic cases could be affected also with limited data about the underlying aetiology and pathogenesis.

Microalbuminuria which is defined as the urinary albumin excretion of 30 $300 \mathrm{mg} / 24 \mathrm{~h}$ has been reported to be a risk factor for renal and cardiovascular disorders [6]; it also has independent correlation to high mortality in diabetic and hypertensive patients [7] [8].

Microalbuminuria has been linked with increased cardiovascular hazards inasymptomatic patients with type 2 DM [9] [10]. On the other hand, an association of obesity with microalbuminuria has also been reported [11]. It has been figured out that the visceral adipose tissue was significantly related to the highest prevalence of microalbuminuria, even in subjects without diabetes [12]. However, the data about the microalbuminuria among non obese, non diabetics patients with NAFLD are limited. 
Aim of the work: Evaluation of urinary albumin/creatinine ratio in non-obese non-diabetic patients with NAFLD.

Patients and methods: Our study was done in the duration between February 2018 and February 2019 in Sohag University Hospital, with total number of 80 patients with NAFLD that were non diabetic non obese patients; 32 of them were female and 48 of them were males, attending to outpatient GIT clinic complaining of various symptoms.

\section{Exclusion criteria:}

1) $\mathrm{BMI}$ equal or more than $30 \mathrm{~kg} / \mathrm{m}^{2}$ (obesity defined as BMI equal or more than 30).

2) Positive results for HCV antibodies, HBsAg.

3) History of hepatobiliary disease.

4) History of renal disease or CKD.

5) Hypertension, DM, Prediabetes, malignancy.

6) Thyroid disease.

7) Ischemic heart disease

8) Autoimmune or metabolic liver disease.

9) Drug intake e.g.; steroid, oral contraceptive pills, statin... etc.

10) Alcohol intake.

11) Fever or chronic infections.

12) Recent vigorous exercise.

13) Volume overloads diseases and storage disease (pseudosteatosis).

An informed consent was obtained prior to subject recruitment according to our university scientific ethical committee recommendations.

Eligible patients enrolled in this study were subjected to the following:

Full history taking, thorough clinical examination, ECG, abdominal ultrasonography and laboratory investigations (CBC, thyroid function test, CRP, ESR, ANA, S. creatinine, liver enzymes, urine analysis, albumin/creatinine ratio, fasting blood sugar, after 2 h., blood sugar, HCV antibodies, HBs Ag, lipogram).

\section{Anthropometric measurements:}

Height, weight and waist circumference were recorded. Waist circumference is measured by using a tape at the level of umbilicus in standing position by single examiner and recorded in centimeters. Body mass index (BMI) was calculated according to the equation; $\mathrm{BMI}=$ Weight $(\mathrm{kg}) / \mathrm{Height}\left(\mathrm{m}^{2}\right)$.

\section{Ultrasonographic assessment of steatosis:}

Fatty liver on ultrasonography (US) displayed in the grey scale looks brighter relative to the kidney cortex. With the increased accumulation of liver fat, US waves become more attenuated resulting in a decreased visualization of the deeper parts of the liver (diaphragm and hepatic veins). Diagnosis of fatty liver was based on the increased echogenicity of the liver parenchyma as compared to the right kidney's cortex. Visibility and sharpness of the diaphragm and hepatic veins' interface were analyzed as well. Based on these 3 parameters steatosis was further classified into 4 grades: Grade 0, no steatosis (liver and renal cortex of the same echogenicity); Grade 1, mild steatosis: slightly brighter liver as com- 
pared to the renal cortex, clear visualization of diaphragm, and interface of hepatic veins with sharp contours; Grade 2, moderate steatosis: brighter liver with attenuated US beam at deeper parts of the liver, diaphragm, and hepatic veins still visible but with blunted contours; Grade 3, severe steatosis: very bright liver, severe US beam attenuation, diaphragm, or hepatic veins not visible. This classification was adopted and already tested by other investigators [13].

Variable transverse and longitudinal gray scale images of the abdomen were acquired by ultrasound equipment [Toshiba Xario 200] using multifrequency convex array transducer (3 - $5 \mathrm{MH}, \mathrm{PUV}-375 \mathrm{BT})$. Patients were scanned in the supine and left lateral decubitus position, utilizing subcostal and intercostal approaches done by the same sonographer to all patients.

Sonograms were performed under fasting conditions. The time-gain compensation was set to adjust the tissue echogenicity as constant as possible regardless of depth.

Patients included in this study were divided according to ultrasonographic classification of fatty liver to 4 groups stated as Group A; with no steatosis. Group B; mild steatosis, Group C; moderate steatosis, Group D; with severe steatosis (Figures 1-4).

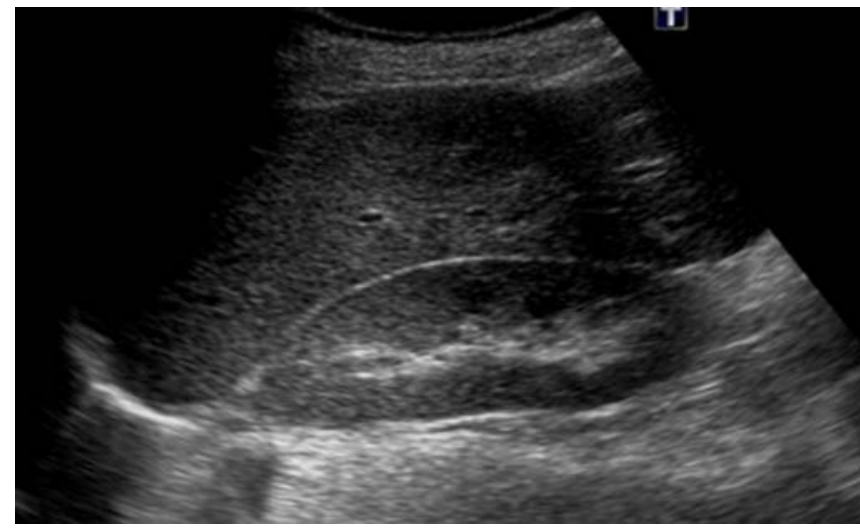

Figure 1. Normal liver parenchyma withsimilar echogenicity as renal cortex.

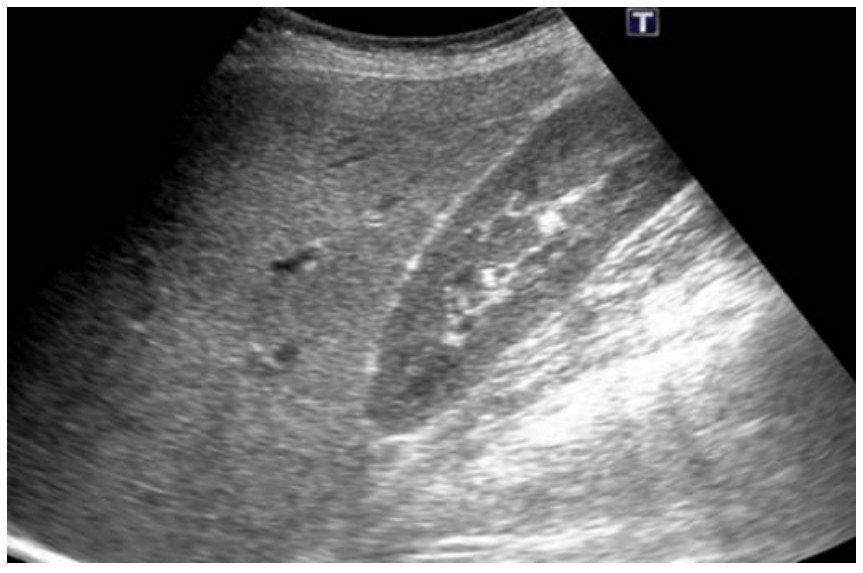

Figure 2. Mild steatosis was defined by a liver echogenicity moderately greater than the right kidney with slight decreased visibility of the intrahepatic vessel walls. 


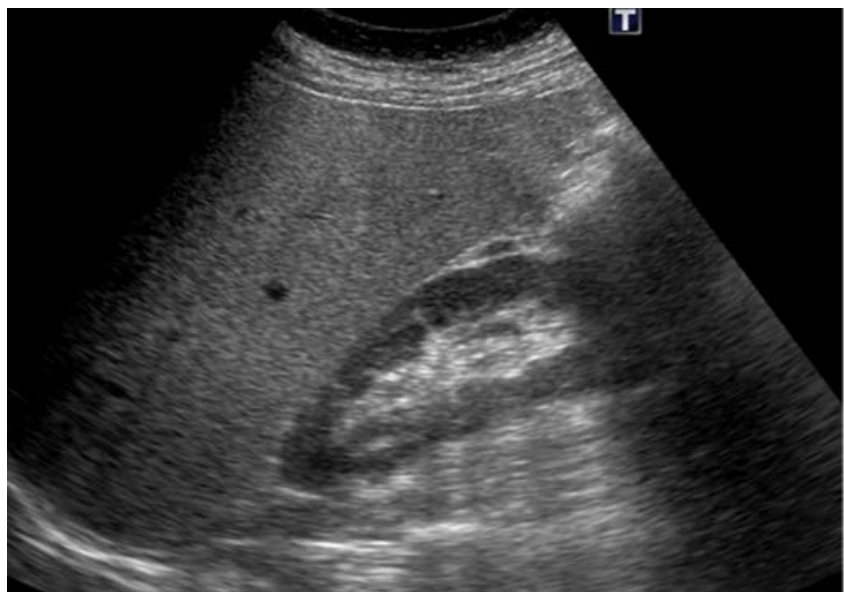

Figure 3. Moderate steatosis was determined by liver echogenicity moderately greater than the right kidney with poor visualization of intrahepatic vessel walls and decreased reflectivity of the hemi diaphragm.

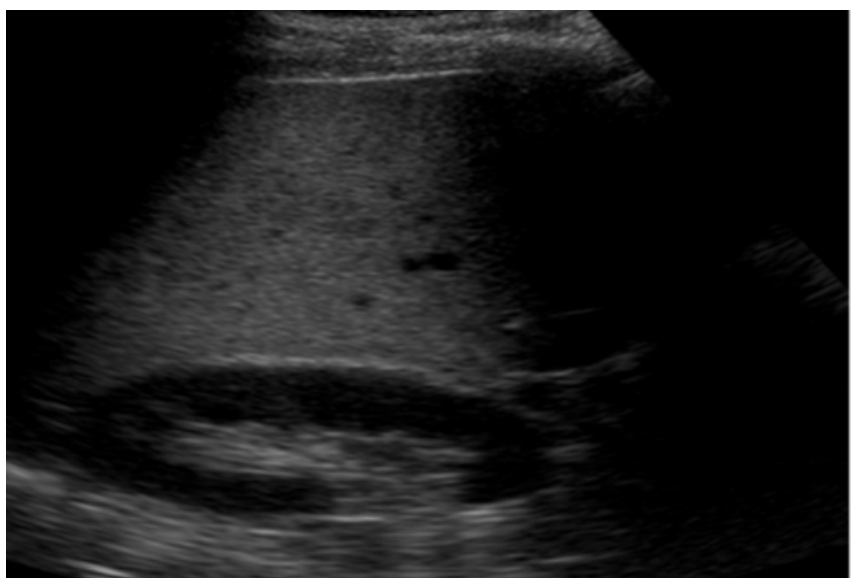

Figure 4. Severe steatosis was determined by a significantly increased echogenicity of the liver compared to the right kidney, a lack of visualization of intrahepatic vessel walls, and markedly decreased reflectivity of the hemidiaphragm.

\section{Laboratory tests:}

Five millimeters of venous blood are collected from each person under aseptic conditions and by using sterile disposable gloves then biochemical assays for alanine aminotransferase (ALT), aspartate aminotransferase (AST), serum creatinine and complete lipid profile (cholesterol, triglyceride, LDL and HDL) were done by using Cobas c311 Chemistry Analyser System (Roche Diagnostic GmbH, Indianopolis, IN, USA). Random urine sample, a random fresh urine sample in early morning is collected in sterile container without using any preservative, followed by centrifugation of the specimen then the following assays are done: micro albumin in urine is measured by using Cobas c311 Chemistry Analyser System (Roche Diagnostic GmbH, Indianopolis, IN, USA) which depend that micro albumin forms a precipitate with a specific antiserum which is determined turbidmetically at $340 \mathrm{~nm}$. Urine creatinine is measured using Cobas c311 Chemistry Analyser System, then we calculate urine albumin (mg/L)/urine creati- 
nine $(\mathrm{gm} / \mathrm{L})$ ratio $=\mathrm{mg}$ albumin $/ \mathrm{g}$ creatinine.

\section{Statistical Analysis}

Data was analyzed using SPSS version 15.0. Quantitative data was expressed as mean \pm standard deviation, median and inter quartile range. Qualitative data was expressed as number and percentage. The data were tested for normality using Shapiro-Wilk test. Kruskal-Wallis test and Spearman's correlation were used for data which wasn't normally distributed.

Chi-Square test was used for comparison between qualitative variables. A 5\% level was chosen as a level of significance in all statistical tests used in the study.

\section{Results}

Eighty non-obese, non-diabetic Subjects ( 32 women, $48 \mathrm{men}$ ) with the mean age of $50.9 \pm 6.1$ years (ranging between 40 and 61 years) were enrolled in the study.

Basic clinical data for the studied population and symptomatology include $32 \mathrm{pa}$ tients (40\%) presented by abdominal pain, 20 patients (25\%) presented by diarrhea, 12 patients (15\%) presented by constipation, 10 patients (12.5\%) presented by nausea and vomiting, and 6 patients (7.5\%) presented by other various symptoms.

The subjects were grouped according to their ultrasound degree of steatosis as follows: control Group (A), (No. 25) 31.25\% of total cases reported as no fat accumulation in liver (no steatosis). Mean BMI was $22.78 \pm 1.69$; mean waist circumference was $81.2 \pm 5$. Group B; No. 21 (26.25\% of total cases reported as had fat accumulation (mild steatosis) stated as (NAFLD 1), mean BMI was $23.34 \pm$ 1.46, mean waist circumference was $82.29 \pm 6.39$. Group C; No. 18 (22.5\%) of total cases and reported as had moderate fat accumulation (moderate steatosis) stated as NAFLD 2, mean BMI was $22.8 \pm 1.76$; mean waist circumference was $82.72 \pm 5.99$.

Group D; No. 16 (20\%) of total cases and reported as had severe fat accumulation (severe steatosis) stated as NAFLD 3, mean BMI was $23.28 \pm 2.92$, mean waist circumference was $85.5 \pm 5.29$.

There was not any statistically significant difference between groups as regards to the age and gender. In evaluation of BMI and waist circumference, with the increasing grade of NAFLD, there was no statistically significant difference between groups as shown in Table 1.

Liver enzymes (ALT, AST) and S creatinine show significant differences between groups despite being in the normal range with no pathological increase in their values, urinary albumin creatinine ratio also show increase in its values with increasing in the degree of steatosis among different groups which is highly statistically significant with $\mathrm{p}$ value less than 0.001 .

Triglycerides, total cholesterol and LDL show also significant changes between groups as they are significantly increase in value as regard increasing in degree of steatosis, inversely noticed with HDL levels as its go down with elevated degree of steatosis which is statistically significant (Table 2, Table 3). 
Table 1. Comparison between the four studied groups regarding age, gender and clinical characteristics.

\begin{tabular}{|c|c|c|c|c|c|}
\hline \multirow[b]{2}{*}{ Variables } & \multicolumn{4}{|c|}{ Group } & \multirow[b]{2}{*}{$P$-value } \\
\hline & $\begin{array}{l}\text { Control Group (A) } \\
\quad(\mathrm{n}=25)\end{array}$ & $\begin{array}{c}\text { Group B (NAFLD 1) } \\
\qquad(\mathrm{n}=21)\end{array}$ & $\begin{array}{c}\text { Group C (NAFLD 2) } \\
\qquad(\mathrm{n}=18)\end{array}$ & $\begin{array}{c}\text { Group D (NAFLD 3) } \\
(\mathrm{n}=16)\end{array}$ & \\
\hline $\begin{array}{c}\text { Age (years) } \\
\text { Mean } \pm \text { S.D. } \\
\text { Median (IQ range) }\end{array}$ & $\begin{array}{c}49.8 \pm 6.88 \\
50(43.5-55)\end{array}$ & $\begin{array}{c}51.24 \pm 7.59 \\
52(43.5-58.5)\end{array}$ & $\begin{array}{c}52.33 \pm 7.27 \\
51.5(46.75-60)\end{array}$ & $\begin{array}{c}50.38 \pm 6.55 \\
49.5(43.75-57.25)\end{array}$ & 0.671 \\
\hline $\begin{array}{l}\text { Gender } \\
\text { Female } \\
\text { Male }\end{array}$ & $\begin{array}{l}11(44 \%) \\
14(56 \%)\end{array}$ & $\begin{array}{c}8(38.1 \%) \\
13(61.9 \%)\end{array}$ & $\begin{array}{c}7(38.9 \%) \\
11(61.1 \%)\end{array}$ & $\begin{array}{c}6(37.5 \%) \\
10(62.5 \%)\end{array}$ & 0.969 \\
\hline $\begin{array}{c}\text { BMI } \\
\text { Mean } \pm \text { S.D. } \\
\text { Median (IQ range) }\end{array}$ & $\begin{array}{c}22.78 \pm 1.69 \\
23.2(21.1-24.55)\end{array}$ & $\begin{array}{c}23.34 \pm 1.46 \\
23.5(22.9-24.3)\end{array}$ & $\begin{array}{c}22.8 \pm 1.76 \\
22.9(20.93-24)\end{array}$ & $\begin{array}{c}23.28 \pm 2.92 \\
22.95(20.68-25.58)\end{array}$ & 0.783 \\
\hline $\begin{array}{c}\text { Waist circumference } \\
\text { Mean } \pm \text { S.D. } \\
\text { Median (IQ range) }\end{array}$ & $\begin{array}{c}81.2 \pm 5 \\
82(79-85)\end{array}$ & $\begin{array}{l}82.29 \pm 6.39 \\
83(76-87)\end{array}$ & $\begin{array}{c}82.72 \pm 5.99 \\
83(79-88.25)\end{array}$ & $\begin{array}{c}85.5 \pm 5.29 \\
87(79.25-89.75)\end{array}$ & 0.195 \\
\hline
\end{tabular}

P-value was calculated by Kruskal Wallis test.

Table 2. Comparison between the four studied groups regarding laboratory characteristics.

\begin{tabular}{|c|c|c|c|c|c|}
\hline \multirow[b]{2}{*}{ Variables } & \multicolumn{4}{|c|}{ Group } & \multirow[b]{2}{*}{ P-value } \\
\hline & $\begin{array}{l}\text { Control Group (A) } \\
\quad(\mathrm{n}=25)\end{array}$ & $\begin{array}{l}\text { Group B (NAFLD 1) } \\
(\mathrm{n}=21)\end{array}$ & $\begin{array}{l}\text { Group C (NAFLD 2) } \\
\qquad(\mathrm{n}=18)\end{array}$ & $\begin{array}{l}\text { Group D (NAFLD 3) } \\
\qquad(\mathrm{n}=16)\end{array}$ & \\
\hline \multicolumn{6}{|l|}{ ALT (U/ml) } \\
\hline Mean \pm S.D. & $21.86 \pm 7.27$ & $25.84 \pm 6.09$ & $26.5 \pm 6.43$ & $30.22 \pm 7.45$ & 0.012 \\
\hline Median (IQ range) & $19(15-28)$ & $25(22-29)$ & $27.5(19.75-30.5)$ & $30.5(25-38)$ & \\
\hline $\begin{array}{c}\text { AST }(\mathrm{U} / \mathrm{ml}) \\
\text { Mean } \pm \text { S.D. } \\
\text { Median (IQ range) }\end{array}$ & $\begin{array}{c}27.86 \pm 2.69 \\
29(25-29.5)\end{array}$ & $\begin{array}{l}30.4 \pm 5.72 \\
29(28-36)\end{array}$ & $\begin{array}{c}34.5 \pm 4.56 \\
35.5(29.5-38.5)\end{array}$ & $\begin{array}{l}34.94 \pm 6.21 \\
38(29-39)\end{array}$ & $<0.001$ \\
\hline \multicolumn{6}{|l|}{ Creatinine (mg/dL) } \\
\hline Mean \pm S.D. & $0.82 \pm 0.19$ & $0.88 \pm 0.19$ & $0.94 \pm 0.15$ & $0.99 \pm 0.13$ & 0.02 \\
\hline Median (IQ range) & $0.8(0.6-0.9)$ & $0.9(0.7-1.05)$ & $0.9(0.88-1.03)$ & $0.95(0.9-1.1)$ & \\
\hline \multicolumn{6}{|l|}{ Albumin/creatinine ratio } \\
\hline Mean \pm S.D. & $16.92 \pm 4.38$ & $23.87 \pm 9.28$ & $25.86 \pm 13.32$ & $32.42 \pm 9.63$ & $<0.001$ \\
\hline Median (IQ range) & $17.9(12.1-19.75)$ & $20.3(16.95-30.7)$ & $20(16.4-41.1)$ & $33(23.28-40.5)$ & \\
\hline \multicolumn{6}{|l|}{ Triglycerides } \\
\hline Mean \pm S.D. & $130.24 \pm 21.73$ & $156.9 \pm 14.96$ & $160.67 \pm 16.62$ & $193.81 \pm 28.66$ & $<0.001$ \\
\hline Median (IQ range) & $130(112.5-145)$ & $160(145-165)$ & $160.5(143.75-175)$ & $192.5(169.5-205)$ & \\
\hline \multicolumn{6}{|l|}{ Total cholesterol } \\
\hline Mean \pm S.D. & $168.64 \pm 8.04$ & $175.67 \pm 29.6$ & $182.06 \pm 16.79$ & $197.5 \pm 24.56$ & $<0.001$ \\
\hline Median (IQ range) & $165(165-172.5)$ & $180(167.5-195)$ & $185(165.75-195)$ & $192.5(175-208.75)$ & \\
\hline \multicolumn{6}{|l|}{ LDL } \\
\hline Mean \pm S.D. & $107.6 \pm 7.79$ & $117.14 \pm 11.39$ & $119.33 \pm 10.63$ & $132.19 \pm 14.83$ & $<0.001$ \\
\hline Median (IQ range) & $105(100-112.5)$ & $120(107.5-125)$ & $121(112.5-125)$ & $132.5(116.25-145)$ & \\
\hline \multicolumn{6}{|l|}{ HDL } \\
\hline Mean \pm S.D. & $39.52 \pm 1.94$ & $37.9 \pm 3.13$ & $36.94 \pm 2.46$ & $33.88 \pm 3.32$ & $<0.001$ \\
\hline Median (IQ range) & $40(38-41)$ & $39(34.5-40)$ & $36.5(35-39)$ & $33.5(30.5-36.5)$ & \\
\hline
\end{tabular}

P-value was calculated by Kruskal Wallis test. P-value $<0.05$ is statistically significant. 
Table 3. Correlation between albumin/creatinine ratio and lipogram among the studied patients.

\begin{tabular}{ccc}
\hline \multirow{2}{*}{ Lipogram } & \multicolumn{2}{c}{ Albumin/creatinine ratio } \\
\cline { 2 - 3 } & $\mathbf{r}$ & P-value \\
\hline Triglycerides & 0.442 & $<0.001$ \\
Total cholesterol & 0.546 & $<0.001$ \\
LDL & 0.49 & $<0.001$ \\
HDL & -0.423 & $<0.001$ \\
\hline
\end{tabular}

$\mathrm{r}=$ Spearman correlation coefficient; $\mathrm{P}$-value $<0.05$ is statistically significant.

A positive correlation between triglycerides, total cholesterol, LDL levels and albumin/creatinine ratio is shown in this table, and negative correlation between HDL and albumin/creatinine ratio with statistically significant values.

\section{Discussion}

The relationship between microalbuminuria and NAFLD has already been established in an obese population or in a diabetic population or both.

The principal objective of this study was to evaluate the relationship between non alcoholic fatty liver disease and microalbuminuria, the results of this study indicate that micro albuminuria is more obvious among NAFLD cases compared with control cases and severity of micro albuminuria was increasing with the advanced stages of NAFLD although two main etiologic factors of microalbuminuria; type 2 diabetes and obesity were excluded.

However, the relationship between microalbuminuria and NAFLD in non obese non diabetic patients was not clear for a population with a traditionally low cardiovascular risk, such as subjects without diabetes or the metabolic syndrome, in this study, even in non diabetic non obese individuals, a significant correlation between degrees of NAFLD and the severity of microalbuminuria was observed.

In our study urinary albumin creatinine ratio show increase in its values with increasing in the degree of steatosis among different groups which was highly statistically significant, those patients with microalbuminuria are risky for chronic kidney disease (CKD) and cardiovascular disease (CVD) in the future and should have a scheduled assessment programs for follow up to avoid disease progression [14] [15].

Triglycerides, total cholesterol and LDL show also significant changes between groups as they are significantly increase in value as regard increasing in degree of steatosis, inversely noticed with HDL levels as it is go down with elevated degree of steatosis which is statistically significant.

Lipid accumulation in cells has been associated with cellular injury and dysfunction [16] [17].

Whether excess lipids are a cause or a sequelae of cell injury has not been universally established, but a lot of evidences suggests that some tissues and cell 
types, lipid overload can be deleterious in a process termed lipotoxicity that may lead to multiple organ dysfunction, involving the heart, endocrine pancreas, skeletal muscle, liver, and the kidney [18].

Moorhead et al. first outlined the lipid nephrotoxicity hypothesis in 1982, proposing that dyslipidemia may contribute to the progression of renal disease. Dyslipidemia could be triggered by urinary albumin loss leading to a compensatory increase in hepatic lipoprotein synthesis and could be part of a positive feedback loop causing further renal injury [19]. Dyslipidemia may directly affect the kidney by causing deleterious renal lipid disturbances (renal lipotoxicity), as well as indirectly through systemic inflammation and oxidative stress, vascular injury, with hormonal changes and other cytokines with renal effect [20] [21].

CKD still remains a major health problem for two reasons. It often goes unrecognized, and the current therapeutic lines have limited effectiveness in retarding disease progression. NAFLD is the most common chronic liver disease and, given the lack of an effective treatment, is becoming the leading indication for liver transplantation in the Western world [22].

NAFLD and CKD is characterized by a deranged cellular substrate metabolism; ectopic fat deposition, which triggers oxidative stress; and inflammatory and profibrotic responses to drive the progression of both disease processes, that may lead to mutual therapeutic strategies. Besides shared pathogenic mechanisms that promote both liver and kidney injury, the fatty liver per se may promote kidney injury and vice versa [23] [24].

Independently of traditional risk factors, the correlation between the severity of NAFLD and the presence and stage of CKD cannot be neglected with shared therapeutic needs and targets in these two conditions.

This may reflect the role of dietary and pharmacological management (i.e. statins) of dyslipidemia in control of microalbuminuria associated with NAFLD.

\section{Conclusion}

After exclusion of type $2 \mathrm{DM}$ and obesity, we conclude that the presence and the severity of microalbuminuria are more apparent among NAFLD patients.

\section{Recommendation}

We recommend that microalbuminuria should be evaluated among non obese non diabetic patients with NAFLD especially with advanced stages and if present; patients should have a strict follow up strategy to avoid and early management of CKD and cardiovascular diseases.

There are some limitations of this study that should be mentioned. First of all the diagnosis and staging of fatty liver disease was based on the findings of ultrasound instead of biopsy. Secondly, glomerular filtration rate was calculated with the MDRD equation instead of a direct measurement since this estimation facilitates the recognition, evaluation, and management of chronic renal disease.

Limitations of this study that should be mentioned: ultrasonography was the 
main tool for diagnosis and staging of fatty liver however biopsy should be done for better evaluation.

\section{Conflicts of Interest}

The authors declare no conflicts of interest regarding the publication of this paper.

\section{References}

[1] Targher, G., Marra, F. and Marchesini, G. (2008) Increased Risk of Cardiovascular Disease in Non-Alcoholic Fatty Liver Disease: Causal Effect or Epiphenomenon? Diabetologia, 51, 1947-1953. https://doi.org/10.1007/s00125-008-1135-4

[2] Vuppalanchi, R. and Chalasani, N. (2009) Nonalcoholic Fatty Liver Disease and Nonalcoholic Steatohepatitis: Selected Practical Issues in Their Evaluation and Management. Hepatology, 49, 306-317. https://doi.org/10.1002/hep.22603

[3] Rodriguez-Hernandez, H., Gonzalez, J.L., Marquez-Ramirez, M.D., et al. (2008) Risk Factors Associated with Nonalcoholic Fatty Liver Disease and Its Relationship with the Hepatic Histological Changes. European Journal of Gastroenterology \& Hepatology, 20, 399-403. https://doi.org/10.1097/MEG.0b013e3282f448af

[4] Sookoian, S. and Pirola, C.J. (2008) Non-Alcoholic Fatty Liver Disease Is Strongly Associated with Carotid Atherosclerosis: A Systematic Review. Journal of Hepatology, 49, 600-607. https://doi.org/10.1016/j.jhep.2008.06.012

[5] Karakurt, F., Carlioglu, A., Koktener, A., et al. (2009) Relationship between Cerebral Arterial Pulsatility and Carotid Intima Media Thickness in Diabetic and Non-Diabetic Patients with Non-Alcoholic Fatty Liver Disease. Journal of Endocrinological Investigation, 32, 63-68. https://doi.org/10.1007/BF03345681

[6] Washio, M., Tokunaga, S., Yoshimasu, K., et al. (2004) Role of Prehypertension in the Development of Coronary Atherosclerosis in Japan. Journal of Epidemiology, 14, 57-62. https://doi.org/10.2188/jea.14.57

[7] Scrutinio, D., Passantino, A., Santoro, D. and Catanzaro, R. (2011) The Cardiorenal Anaemia Syndrome in Systolic Heart Failure: Prevalence, Clinical Correlates, and Long-Term Survival. European Journal of Heart Failure, 13, 61-67. https://doi.org/10.1093/eurjhf/hfq167

[8] Arnlov, J., Evans, J.C., Meigs, J.B., et al. (2005) Low-Grade Albuminuria and Incidence of Cardiovascular Disease Events in Nonhypertensive and Nondiabetic Individuals: The Framingham Heart Study. Circulation, 112, 969-975. https://doi.org/10.1161/CIRCULATIONAHA.105.538132

[9] Currie, G., McKay, G. and Delles, C. (2014) Biomarkers in Diabetic Nephropathy: Present and Future. World Journal of Diabetes, 5, 763-776. https://doi.org/10.4239/wjd.v5.i6.763

[10] Kim, J.J., Hwang, B.H., Choi, I.J., et al. (2015) A Prospective Two-Center Study on the Associations between Microalbuminuria, Coronary Atherosclerosis and Long-Term Clinical Outcome in Asymptomatic Patients with Type 2 Diabetes Mellitus: Evaluation by Coronary CT Angiography. The International Journal of Cardiovascular Imaging, 31, 193-203. https://doi.org/10.1007/s10554-014-0541-6

[11] Du, N., Peng, H., Chao, X., et al. (2014) Interaction of Obesity and Central Obesity on Elevated Urinary Albumin-to-Creatinine Ratio. PLoS ONE, 9, e98926. https://doi.org/10.1371/journal.pone.0098926

[12] Kim, H., Kim, H.J., Shin, N., et al. (2014) Visceral Obesity Is Associated with Mi- 
croalbuminuria in Nondiabetic Asians. Hypertension Research, 37, 679-684. https://doi.org/10.1038/hr.2014.47

[13] Van Werven, J.R., Marsman, H.A., Nederveen, A.J., et al. (2010) Assessment of Hepatic Steatosis in Patients Undergoing Liver Resection: Comparison of US, CT, T1-Weighted Dual-Echo MR Imaging, and Point-Resolved 1H MR Spectroscopy. Radiology, 256, 159-168. https://doi.org/10.1148/radiol.10091790

[14] Stehouwer, C.D.A. and Smulders, Y.M. (2006) Microalbuminuria and Risk for Cardiovascular Disease: Analysis of Potential Mechanisms. JASN, 17, 2106-2111. https://doi.org/10.1681/ASN.2005121288

[15] Sarafidis, P.A. and Bakris, G.L. (2006) Microalbuminuria and Chronic Kidney Disease as Risk Factors for Cardiovascular Disease. Nephrology Dialysis Transplantation, 21, 2366-2374. https://doi.org/10.1093/ndt/gfl309

[16] Weinberg, J.M. (2006) Lipotoxicity. Kidney International, 70, 1560-1566. https://doi.org/10.1038/sj.ki.5001834

[17] Unger, R.H., Clark, G.O., Scherer, P.E., et al. (2010) Lipid Homeostasis, Lipotoxicity and the Metabolic Syndrome. Biochimica et Biophysica Acta, 1801, 209-214. https://doi.org/10.1016/j.bbalip.2009.10.006

[18] Schaffer, J.E. (2003) Lipotoxicity: When Tissues Overeat. Current Opinion in Lipidology, 14, 281-287. https://doi.org/10.1097/00041433-200306000-00008

[19] Moorhead, J.F., Chan, M.K., El-Nahas, M., et al. (1982) Lipid Nephrotoxicity in Chronic Progressive Glomerular and Tubulo-Interstitial Disease. The Lancet, 2, 1309-1311. https://doi.org/10.1016/S0140-6736(82)91513-6

[20] Ruan, X.Z., Varghese, Z. and Moorhead, J.F. (2009) An Update on the Lipid Nephrotoxicity Hypothesis. Nature Reviews Nephrology, 5, 713-721. https://doi.org/10.1038/nrneph.2009.184

[21] Wahba, I.M. and Mak, R.H. (2007) Obesity and Obesity-Initiated Metabolic Syndrome: Mechanistic Links to Chronic Kidney Disease. Clinical Journal of the American Society of Nephrology, 2, 550-562. https://doi.org/10.2215/CJN.04071206

[22] Singal, A.K., Salameh, H., Kuo, Y.F. and Wiesner, R.H. (2014) Evolving Frequency and Outcomes of Simultaneous Liver Kidney Transplants Based on Liver Disease Etiology. Transplantation, 98, 216-221. https://doi.org/10.1097/TP.0000000000000048

[23] Musso, G., Cassader, M. and Gambino, R. (2016) Nonalcoholic Steatohepatitis: Emerging Molecular Targets and Therapeutic Strategies. Nature Reviews Drug Discovery, 15, 249-274. https://doi.org/10.1038/nrd.2015.3

[24] Musso, G., et al. (2016) Fatty Liver and Chronic Kidney Disease: Novel Mechanistic Insights and Therapeutic Opportunities. Diabetes Care, 39, 1830-1845.

https://doi.org/10.2337/dc15-1182 